INPLASY

PROTOCOL

To cite: Gao et al. A Metaanalysis of Clinical Efficacy of Extracorporeal Shock Wave for Plantar Fasciitis. Inplasy protocol 202150093. doi: 10.37766/inplasy2021.5.0093

Received: 26 May 2021

Published: 26 May 2021

Corresponding author: Gao Ning

gaoning0906@163.com

Author Affiliation: Hunan University Of Technology

Support: Hunan Key Laboratory.

Review Stage at time of this submission: The review has not yet started.

Conflicts of interest: None declared.

\section{A Meta-analysis of Clinical Efficacy of Extracorporeal Shock Wave for Plantar Fasciitis}

Gao, N1; Zhang, Qy2; Wang, Sq33 Wang, Gj4.

Review question / Objective: P: Plantar Fasciitis I: Extracorporeal Shock Wave C: Other therapy O: VAS; Plantar fascia thickness; Maximum walking walk S: RCTs. Condition being studied: 1) were patients with plantar fasciitis and diagnosed as plantar fasciitis based on medical history and physical examination; 2) A randomized controlled experiment (RCT) on clinical efficacy of plantar fasasitis in vitro without blind method; 3 ) all subjects must be $\geq 18$ years of age with no gender restriction; 4) at least one test group took simple in vitro shock wave therapy as intervention; 5) selected visual simulation score (VAS) score as the primary outcome index, and plantar fascia thickness and maximum continuous walking time as secondary outcome indicators.

INPLASY registration number: This protocol was registered with the International Platform of Registered Systematic Review and Meta-Analysis Protocols (INPLASY) on 26 May 2021 and was last updated on 26 May 2021 (registration number INPLASY202150093).

\section{INTRODUCTION}

Review question / Objective: P: Plantar Fasciitis I: Extracorporeal Shock Wave C: Other therapy O: VAS; Plantar fascia thickness; Maximum walking walk $S$ : RCTs.
Condition being studied: 1) were patients with plantar fasciitis and diagnosed as plantar fasciitis based on medical history and physical examination; 2) A randomized controlled experiment (RCT) on clinical efficacy of plantar fasasitis in vitro without blind method; 3) all subjects must be $\geq 18$ 
years of age with no gender restriction; 4) at least one test group took simple in vitro shock wave therapy as intervention; 5) selected visual simulation score (VAS) score as the primary outcome index, and plantar fascia thickness and maximum continuous walking time as secondary outcome indicators.

\section{METHODS}

Participant or population: Plantar Fasciitis.

Intervention: Extracorporeal Shock Wave.

Comparator: Other therapy.

Study designs to be included: RCTs.

Eligibility criteria: 1) were patients with plantar fasciitis and diagnosed as plantar fasciitis based on medical history and physical examination; 2) A randomized controlled experiment (RCT) on clinical efficacy of plantar fasasitis in vitro without blind method; 3 ) all subjects must be $\geq 18$ years of age with no gender restriction; 4) at least one test group took simple in vitro shock wave therapy as intervention; 5) selected visual simulation score (VAS) score as the primary outcome index, and plantar fascia thickness and maximum continuous walking time as secondary outcome indicators.

Information sources: Computer retrieval was conducted in six databases, including PubMed, Cochrane Library, Embase, CNKI, VIP and Wanfang. The retrieval time was restricted from the establishment of the database to March 2021.

Main outcome(s): A total of 16 literatures were included, including 1198 subjects, including 601 in the extracorporeal shock wave group and 597 in the control group.

Quality assessment / Risk of bias analysis: The bias assessment method was the Cochrane bias risk assessment tool, and the statistical software was Review Manager 5.3 and Stata14.0.
Strategy of data synthesis: Search for a randomized controlled trial (RCT) of extracorporeal shock wave therapy for plantar fasciitis. The included studies were independently screened by two investigators and features were extracted.

Subgroup analysis: RCTs.

Sensitivity analysis: Stata 14.0 for sensitivity analysis and no Meta analysis results after each 1 literature, indicating stable results.

Country(ies) involved: China.

Keywords: Plantar fasciitis; Extracorporeal shock wave; RCT; Meta analysis.

Contributions of each author:

Author 1 - Gao Ning.

Email: gaoning0906@163.com

Author 2 - Zhang Qy.

Author 3 - Wang Sq.

Author 4 - Wang Gj.

Email: wgj_1213@aliyun.com 